\title{
Performance evaluation of flexible cross correlation (FCC) OCDMA code based on radio over fiber (RoF) simulation system
}

\author{
Z.Ibrahim, C.B.M.Rashidi, S.A.Aljunaid, A.K.Rahman, M.S.Anuar \\ School of Computer and Communication, University Malaysia Perlis (UniMAP), Malaysia
}

\begin{tabular}{|c|c|}
\hline Article Info & ABSTRACT \\
\hline Article history: & This paper investigates the performance of an elegance code set algorithm \\
\hline & known as Flexible Cross Correlation (FCC) code to be run into a simulation \\
\hline Received Sep 23, 2018 & test of proposed RoF system. Executing OCDMA Code into RoF framework \\
\hline Revised Nov 24, 2018 & is seen as a promising technique for enhancing spectral efficiency and \\
\hline Accepted Dec 8, 2018 & $\begin{array}{l}\text { meanwhile enhanced the length of fiber optic cable and data bit rate } \\
\text { execution while reduce the passive equipment device. This code can }\end{array}$ \\
\hline Keywords: & $\begin{array}{l}\text { efficaciously reduce the results of phase-induced intensity noise (PIIN), it has } \\
\text { the multiple access interference (MAI) cancellation belongs to RoF system }\end{array}$ \\
\hline Bit error rate (BER) & $\begin{array}{l}\text { and at the same time simple and easy within the code construction. Bit error } \\
\text { rate (BER) performance is assessed for this proposed simulation system }\end{array}$ \\
\hline Data bit rate & The evaluation made based on the performance of fiber length, data bit rate, \\
\hline FCC-OCDMA & related with Bit Error Rate (BER) and also the complexity of the system. \\
\hline OCDMA-RoF & $\begin{array}{l}\text { The extensive simulation results reveal FCC code possibly will accomplish } \\
\text { sufficiently up to } 51 \mathrm{~km} \text { at BER } 10-9 \text { for data rate } 155 \mathrm{Mbps} \text { as matched to } \\
622 \text { Mbps with exponentially increases at BER } 10-12 \text { error floor and it is fit } \\
\text { for short haul networking such as FTTH and local area network (LAN). }\end{array}$ \\
\hline
\end{tabular}

Copyright $@ 2019$ Institute of Advanced Engineering and Science. All rights reserved.

\section{Corresponding Author:}

Ibrahim Zakaria,

School of Computer and Communication Engineering,

University Malaysia Perlis (UniMAP),

1st Floor, Pauh Putra Main Campus, 02600 Arau, Perlis Malaysia.

Email: ibrahim_im90@yahoo.com

\section{INTRODUCTION}

Tremendous enthusiasm for interactive media data, higher data speeds, for top notch video and a growing number of clients; are putting the burden on wireless communication system merchants to offer higher data rates. Reduce this pressure could be refined by utilizing a microcellular system, which infers lessening the cell size, and thusly, it might diminish the power utilization of the mobile station [1].

By using an optical fiber to partner central station (CS) with numerous remote, it can simplify the base stations (BS), it can essentially grow the system capacity, coverage, and portability what's more serving both fixed and mobile end clients [2]. The RoF offers a combination of high capacity optical fiber and adaptability wireless systems. This advancement is displayed to reduce framework cost and the intricacy of remote antenna units (RAU). Moreover, RoF advancement is a contender to update the execution of wireless communication system with the blend of extensive bandwidth (BW) and low attenuation qualities offered by optical fiber [2].

Many codes have been proposed for optical CDMA framework [3], [4]. Be that as it may, just a couple of them [5], [6] have been appeared to really bolster multi-rate transmissions. The multi-rate transmission is one of the principle points of interest of OCDMA system which give asynchronous transmissions instead of the synchronous time division multiplexing (TDM) frameworks. The FCC code gives basic tridiagonal matrix developments contrasted with the other SAC-OCDMA codes, for example, 
MDW, MQC and MFH codes. FCC code has such a different preferred standpoint, including the less demanding code development, less many-sided quality of encoder/decoder plan and adaptable in-phase crosscorrelation for uncomplicated to execute utilizing Fiber Bragg Gratings (FBGs) for the OCDMA frameworks [7]. In this paper, we have executed FCC code into proposed Radio over Fiber (ROF) framework to investigate the execution through simulation system apparatus.

\section{FCC OCDMA CODE CONSTRUCTION}

Optical codes are the group of $K$ (for $K$ users) binary [0,1] arrangements of length $N$, code weight $W$ (the number of " $1 "$ in each code word) and the most extreme cross-connection, $\lambda_{\max }$. In OCDMA framework, optical codes ought to have substantial estimations of $W$ and the size $K$ to permit receivers to separate each of the conceivable users, to lesser channel interference and furthermore to suit the huge number of users.

\subsection{First Step}

The set optical code consists of $\left(N, W, \lambda_{\max }\right)$ FCC code for $K$ users. The $K \times N$ code matrix $A_{K}^{W}$ is here called the Tridiagonal Code Matrix. These arrangements of codes are then written as [8];

$$
A_{K}^{W}=\left[\begin{array}{ccccccc}
a_{11} & a_{12} & a_{13} & 0 & 0 & \cdots & 0 \\
a_{21} & a_{22} & a_{23} & a_{24} & 0 & \cdots & \vdots \\
0 & a_{32} & a_{33} & a_{34} & a_{35} & 0 & \vdots \\
0 & 0 & a_{43} & a_{44} & a_{45} & a_{46} & \vdots \\
\vdots & \ddots & \ddots & \ddots & \ddots & \ddots & \vdots \\
0 & 0 & \cdots & \cdots & \cdots & \cdots & a_{K N}
\end{array}\right]=\left[\begin{array}{c}
A_{1} \\
A_{2} \\
A_{3} \\
\vdots \\
\vdots \\
A_{K}
\end{array}\right]
$$

Where,

$$
\begin{gathered}
A_{1}=a_{11}, a_{12}, a_{13} \ldots a_{1 N} \\
A_{2}=a_{21}, a_{22}, a_{23}, a_{24} \ldots a_{2 N} \\
A_{3}=a_{31}, a_{32}, a_{33}, a_{34} \ldots a_{3 N} \\
\vdots \\
A_{K}=a_{K 1}, a_{K 2}, a_{K 3} \ldots a_{K N}
\end{gathered}
$$

The rows of $A_{l}, A_{2}$, and $A_{k}$ speak to the $K$ code word and it is accepted that the cold weight of each of the $K$ code words is to be $W$.

\subsection{Second Step}

After the $K$ codes spoke to by the $K$ rows of the $K \times N$ code matrix $A_{K}^{W}$ in (1), the code is utilized to speak to a legitimate arrangement a valid set of $K$ code word within phase cross-correlations $\lambda_{\max }$ and code weight $W[9]$. The elements $\left\{\mathrm{a}_{\mathrm{ij}}\right\}$ of $A_{K}^{W}$ must have values either " 0 " or " 1 ".

$$
\text { aij = "0"or"1" }\left\{\frac{\text { for }_{-} i=1,2, \ldots, K}{j=1,2, \ldots, N}\right\}
$$

The in phase cross-correlation $\lambda_{\max }$, between any of the $K$ code words ( $K$ rows of the matrix $A_{K}^{W}$ ), should not exceed code weight $W$. That is,

$$
X_{i} X_{j}^{T}= \begin{cases}\leq \lambda_{\max } & \text { for } i \neq j \\ =W & \text { for } i=j\end{cases}
$$

The code weight of each code word should be equal to $\mathrm{W}$ where, 


$$
\sum_{j=1}^{N} a_{i j}=W, i=1,2 \ldots K
$$

As shown in (3), it is seen that the $W=X_{i} X_{i}^{T}$ is the in phase auto-correlation function of codes. $X_{i}$ $Y_{j}^{T}$ is the out of phase cross-correlation between the $i^{\text {th }}$ and the $j^{\text {th }}$ codes. It follows that $X_{i} X_{i}^{T}$ should be greater than $X_{i} Y_{j}^{T}$. In other words, $W>\lambda_{\max }$. All K rows of $A_{K}^{W}$ should be linearly independent because each code word must be uniquely different from other code words. That is to say, the rank of the code matrix $A_{K}^{W}$ should be $K$. Moreover, for $A_{K}^{W}$ to have rank $K$, thus codes $N \geq K$.

\subsection{Third Step}

Out of five conditions above, one of the matrices binary sequences as shown in (1), who's the first $i^{\text {th }}$ row for the first $\mathrm{K}$ user is given by [10];

$$
A_{i}=\begin{array}{ccc}
r(i-1) & W & r(K-i) \\
0.0 & 11 . .1 & 0 \ldots 0
\end{array}
$$

The length $N$ of the codes which is the length of the rows of the $K \times N$ code matrix $A_{K}^{W}$ is given by;

$$
N=W K-\lambda_{\max }(K-1)
$$

It can be seen that the length $N$ is least under the accepted conditions. This technique will now be clarified with the assistance of an illustration; it is sought to generate a set of minimum length with flexible in phase cross-correlation optical code. Table 1 shows the FCC code for a given number of users $K=4$, code weight $W=3$ and flexible cross- correlation $\lambda_{\max } \leq 1$.

Table 1. The FCC Code with $K=4, W=3, \lambda_{\max } \leq 1$ and $N=9$

\begin{tabular}{ccccc}
\hline Wavelengths & $\lambda 1=1550.0$ & $\lambda 1=1550.8$ & $\lambda 1=1551.6$ & $\lambda 1=1552.4$ \\
\hline User 1 & 1 & 1 & 0 & 0 \\
User 2 & 0 & 1 & 1 & 0 \\
User 3 & 0 & 0 & 1 & 1 \\
\hline
\end{tabular}

\section{PROPOSED OCDMA RoF SIMULATION SYSTEM}

Figure 1 shows the block diagram of proposed RoF system executing FCC OCDMA code, two transmitters will be utilized. Each transmitter includes 4 OLTs and 4 ONUs at the receiver, along these lines the aggregate of OLTs for two transmitters will be 8 OLTs. The pseudo-random bit sequence (PRBS) is utilized to generate binary digits randomly which is used to produce NRZ or RZ pulse coded signal. This coded signal represents the data that needed to be transmitted to the receiver. This coded signals required a frequency signal to act as a carrier. In this manner, sine generator is signifying generate an electrical sine waveform frequency signal, keeping in mind the end goal to convey coded signal in the wake of converging with the frequency. Four low intermediate frequencies (IF) are selected according to four OLTs. Each OLT having IF of $1.21 \mathrm{GHz}, 1.81 \mathrm{GHz}, 2.41 \mathrm{GHz}$, and $3.01 \mathrm{GHz}$. Any frequency value ought to be fine as long it is underneath $10 \mathrm{GHz}$. These four electrical carrier signals are combined together using the combiner, which is then formed a subcarrier multiplexed (SCM) signals.

A Mach-Zehnder modulator (MZM) is driven by SCM electrical signals and LED to merge into an optical carrier signal. OCDMA encoder devices exploiting one single broadband source being sliced for three channels with code weight equal to two. An evasion interference subtraction is used as the detection scheme at the receiver. Figure 2 shows the encoder configuration operating the FCC code sequence which offers simplicity and cost-effectiveness consuming the LED as a light source. Each wavelength produce will be used as a carrier for each coded signal. For instance, coded signal having IF of $1.21 \mathrm{GHz}$ will be modulated with wavelength $(\lambda 1)$ of $1550 \mathrm{~nm}$ and vice versa. The Central wavelength of $1550 \mathrm{~nm}$ around C-band $(1525-1565 \mathrm{~nm})$ is popularly known as zero loss window, means silica fiber gives the least attenuation at this wavelength approximately $0.2 \mathrm{~dB} / \mathrm{km}$, which more beneficial to be used as optical carrier signal wavelength. These wavelengths are required to be coupled up together using the multiplexer (MUX) to become one optical band signal. Afterward, the optical band is transmitted through Single Mode Optical Fiber cable. SMF cable is picking contrasted with Multi-Mode Fiber (MMF) cable in light of the fact that, an SMF Performance evaluation of flexible cross correlation (FCC) OCDMA code based on radio over... (Z.Ibrahim) 
proficient to dispose of higher order modes and the attenuation is smaller since less light will encounter absorption and scattering effects. codes are the group of $\mathrm{K}$ (for $\mathrm{K}$ users) binary $[0,1]$ arrangements of length $\mathrm{N}$, code weight $\mathrm{W}$ (the number of " 1 " in each code.

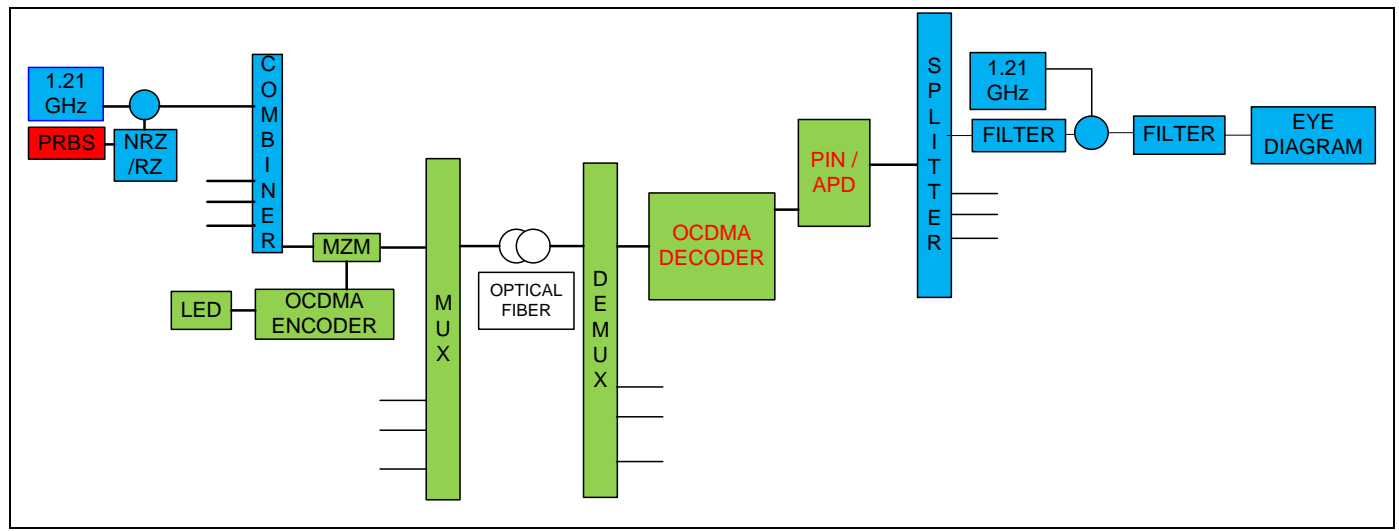

Figure 1. Block diagram of proposed FCC OCDMA-RoF system

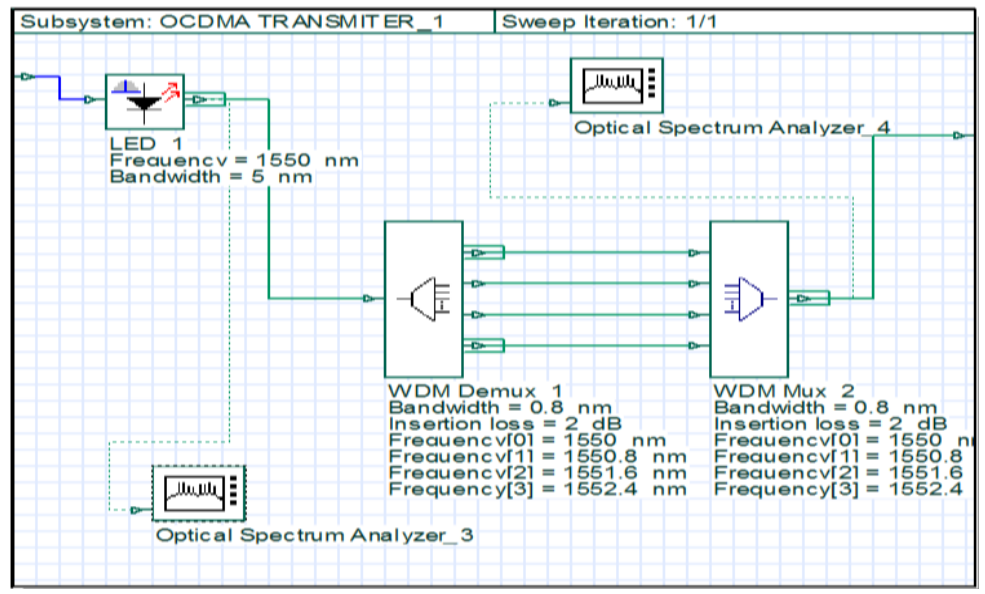

Figure 2. Proposed OCDMA encoder simulation system

Toward the finish of the SMF cable, the optical bands feed to OCDMA decoder employing fiber Bragg grating (FBG) which will decode the acceptance signal. FBG is used to isolate the wavelengths and assign an appropriate time slot to each one [13]. Figure 3 demonstrate three FBG that encode two wavelengths symbolised by $\lambda 2, \lambda 3$, and $\lambda 3, \lambda 1$ which are correlated or overlapping wavelength as shown in Table 1. Due to that, FBG bandwidth will have a spectral width of $1.6 \mathrm{~nm}$ for corresponded wavelength and $0.8 \mathrm{~nm}$ for uncorrelated wavelength such as $\lambda 1$ and $\lambda 4$ for both wavelengths. PIN Photodetector detects and demodulates the wavelengths to transform it once more into an electrical carrier signal that contains data information from the OLTs. These carrier signals are split uniformly by the power splitter which distributes the carrier signals to four ONUs. To permit the exact carrier signals entering each ONUs, Band Pass Bessel Filter is used and filtered the carrier signals alluding the same IF values from OLT.

Once it is filtered, each signal needs to be up-converted to the IF values using coherent detection technique to extricate any phase and frequency information and enormously enhanced the receiver sensitivity. In this way, sine generator is added at each ONU to generate IF and merge with each signal carrier. Later on, the carrier signals once again being filtered by Low Pass Bessel Filter to totally dispense with any signals above the cut-off frequency of the up-conversion process. The most widely recognized parameters utilized for measuring the execution are Q-factor and bit error rate (BER). The BER considered as the probability of inaccurate distinguishing proof of a bit by the choice circuit in the receiver and Q-factor measures the quality of the transmission of signals in terms of its signal-to-noise ratio (SNR), 
higher the approximation of Q-factor, the superior the SNR and in this way, the lower the chance of bit error. BER analyzer is utilized to break down the execution of each ONU in the receiver side. As a result of the FCC OCDMA encoding, the signals which are required by different Base Stations is generally incomprehensible. Interference along the fiber interface and each Base Station could construct a private and secure association with the Central Station.

Analyzer execution for this back to back transmission accomplished limit 10-9 after the transmission over more than 40km SMF. Nevertheless, the fiber transmission is put stock in prepared to reach up to $45 \mathrm{~km}$ for this upgraded design. Figure 4 shows the finished design of proposed FCC OCDMARoF simulation system.

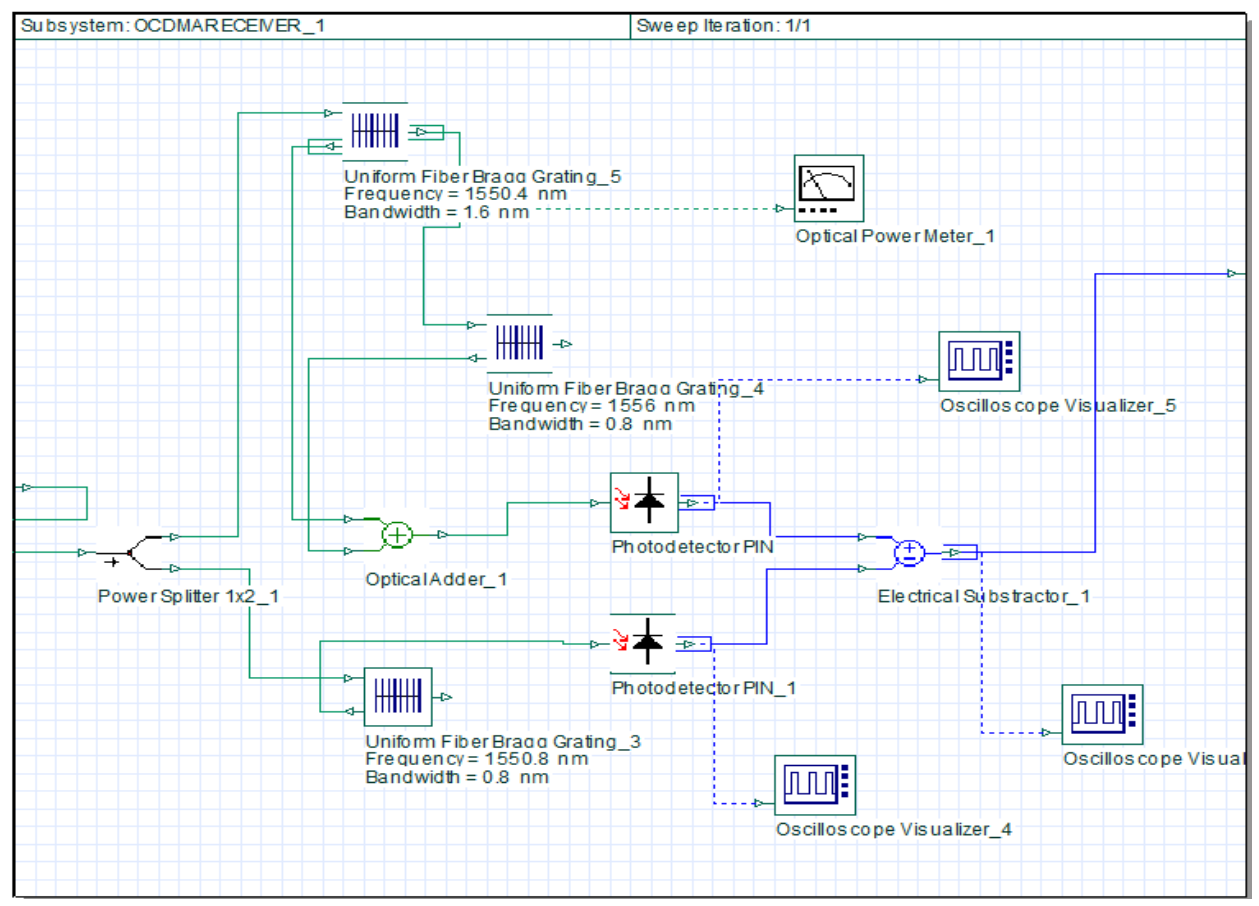

Figure 3. Proposed OCDMA decoder simulation system

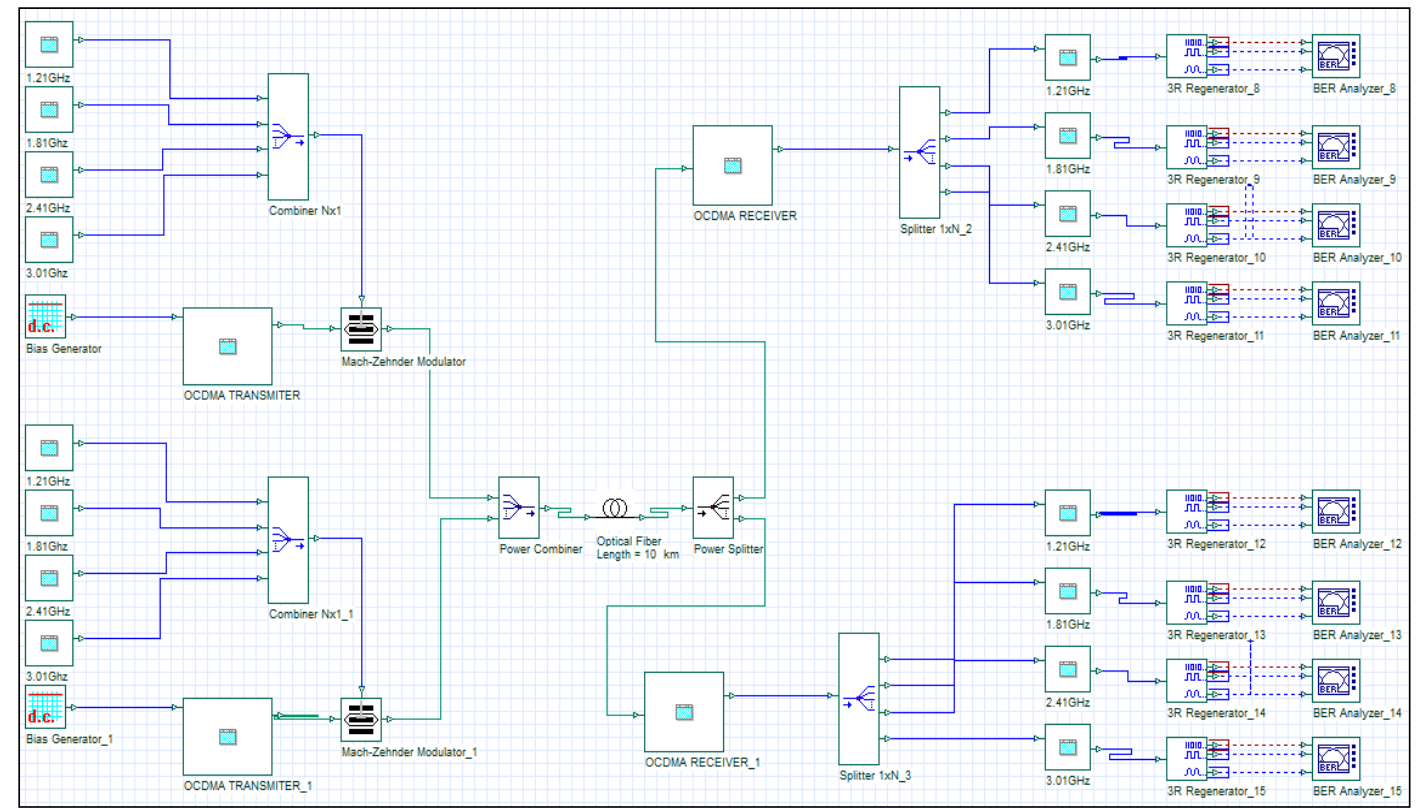

Figure 4. Proposed complete FCC OCDMA-RoF simulation system 


\section{RESULT AND DISCUSSION}

Optical The performance of OCDMA-RoF was demonstrated by using OptiSystem software from Optiwave $^{T M}$ and the simulation parameters are shown in Table 2. The FCC code was implemented in an OCDMA encoder system inside the LED wavelength setting based on the FCC Code wavelength. The simulation parameters, for example, attenuation, dispersion effects and non-linear effects such as fourwave mixing are initiated amid re-enactment and indicated by ordinary industry qualities to mimic the genuine environment as close as would be prudent. The exhibitions of the framework were described by alluding to the received power at various bit rates and also an RF spectrum signal of NRZ format [4]. The performances of the system were characterized by referring to the effect of fiber length on BER performance and the suitable data bit rate for the system for FCC-OCDMA RoF system. The comparison made based on the performance of earlier proposed RoF system (without FCC code) with proposed FCC OCDMA-RoF system.

Table 2. Parameters of the Simulation Analysis

\begin{tabular}{ccccc}
\hline Wavelengths & $\lambda 1=1550.0$ & $\lambda 1=1550.8$ & $\lambda 1=1551.6$ & $\lambda 1=1552.4$ \\
\hline User 1 & 1 & 1 & 0 & 0 \\
User 2 & 0 & 1 & 1 & 0 \\
User 3 & 0 & 0 & 1 & 1 \\
\hline
\end{tabular}

\subsection{Bit Error Rate (BER) Versus Fiber Length}

The Figure 5 demonstrates the plots between fiber length and framework execution BER actualizing FCC Code for bit rates of $155 \mathrm{Mbps}$. The earlier proposed RoF system utilizing laser with one wavelength to carry four data to be transmitted to the receiver. However, utilizing of FCC code, the wavelength of $1550 \mathrm{~nm}$ able to be spliced to another three wavelengths $(1550.8,1551.6$ and $1552.4 \mathrm{~nm})$ for carrying the four data without interfering each other. It can be seen that, at the framework execution BER of $10^{-9}$, RoF system with implementing FCC code certainly has better framework execution BER, where it is fit for accomplishing a worthy BER limit satisfactorily for $51 \mathrm{~km}$ fiber length.

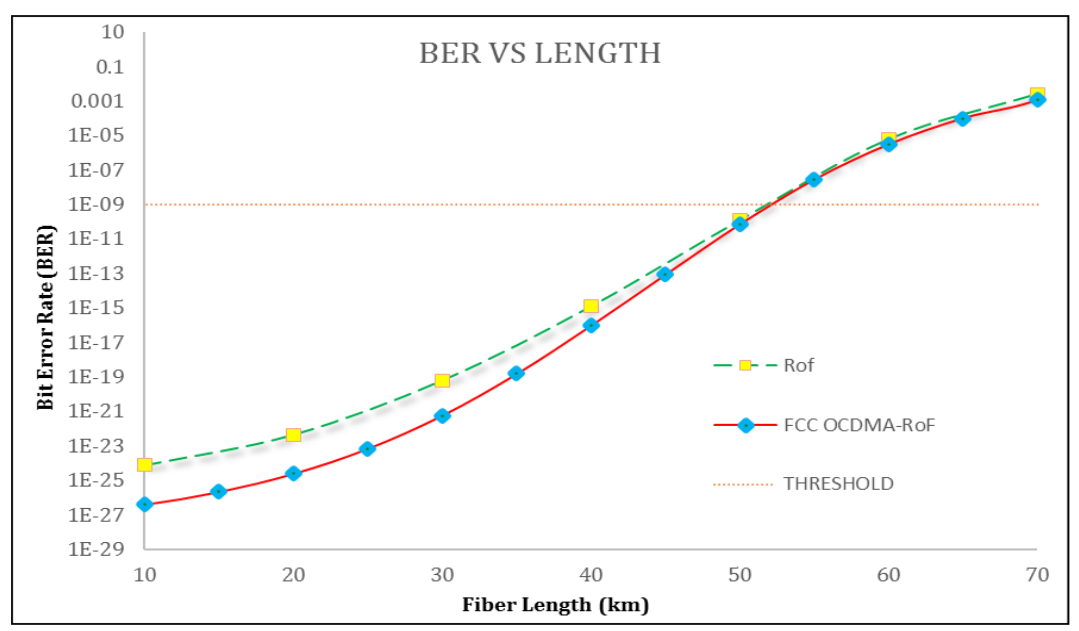

Figure 5. BER performance on fiber length

Meanwhile, the RoF system without implement FCC code achieved $50 \mathrm{~km}$ before the performance decrease. Even though, only small different change within these two system, FCC code help to improve the performance, which able to prevent multiple access interference between the optical signal that carries four data from one transmitter. Furthermore, as the fiber length is expanded more than $50 \mathrm{~km}$, the index of chromatic dispersion of FCC code for the optical fiber increase. In this way, BER execution degrades because of dispersion impacts in the FCC OCDMA framework. To beat the issue, the BER can be lessened by including the chips, while the impact of the chromatic dispersion is decreased by increasing the force of the optical transmitter power. Rather than the execution of the FCC code itself, the execution is additionally related to the proposed RoF framework. RF coherent detection is actualized at the receiving area to extract 
any phase and frequency information, and enormously enhanced the receiver affectability. Thus, this detection technique and FCC code help to increase the fiber length with a minimum BER value.

\subsection{Bit Error Rate (BER) Versus Data Bit Rate}

Figure 6 exhibit the execution FCC code in term of different data bit rate and the framework execution BER tried at $10 \mathrm{~km}$ of optical fiber length. As should be obvious from the graph, the lower the bit rate $(155 \mathrm{Mb} / \mathrm{s})$, the FCC code execution completely increases. The BER accomplished for $155 \mathrm{Mb} / \mathrm{s}$ is roughly around $7.56 \times 10^{-25}$. While expanding the data bit rate to $622 \mathrm{Mb} / \mathrm{s}$, the BER diminished to $1.00 \times 10^{-12}$, which let us know the BER esteem get to be distinctly greater and decrease the execution. Meanwhile, for the RoF system without FCC code, for bit rate $155 \mathrm{Mb} / \mathrm{s}$, the BER achieved at $2.39 \times 10^{-25}$ and as bit rate increase to $622 \mathrm{Mb} / \mathrm{s}$, the BER performance decrease to $5.05 \times 10^{-1}$. The FCC code helps to improve the performance on the proposed RoF system. As the bit rate expanded for both systems to $\mathrm{GB} / \mathrm{s}$ scale $(1 \mathrm{~GB} / \mathrm{s}, 2 \mathrm{~GB} / \mathrm{s}$, and $3 \mathrm{~GB} / \mathrm{s}$ ), the BER values kept on diminishing monotonically. Beginning from $1 \mathrm{~GB} / \mathrm{s}$ to $3 \mathrm{~GB} / \mathrm{s}$, the data bit rate goes with rapid and turn out to be too quick for the framework. It will prompt to a deterioration of the BER behavior. This is going on because of the diminishing of the pulse width as producing the sensitive bits signal to dispersion effect.

FCC code confronting strong non-linear interference suppression for higher bit rate. Expanding the bit rate more than $1 \mathrm{~Gb} / \mathrm{s}$ requires extremely narrow pulses which are troublesome for FCC code to generate and posture challenges in transmission and detection in this manner increasing BER value. Interestingly, the need to perform encoding and decoding for FCC OCDMA simulation postures one quick challenge which as of now methodologies the breaking point of electronic handling due to both the optical carrier frequency and higher bit rate required power scaling. Scaling to suggestively greater bit rates and channel numbers would require a very large optical amplifier at each receiver, which is highly unwelcome for application to networks with large numbers of nodes.

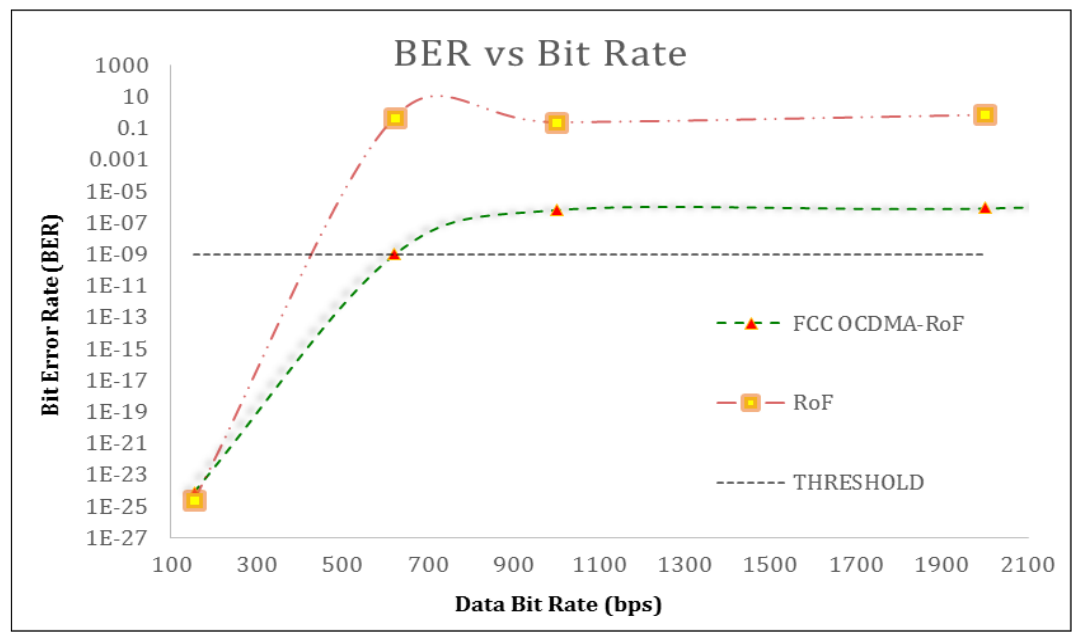

Figure 6. BER performance on various data bit rate

\section{CONCLUSION}

In this research, the work focusing on analysis of the performance of FCC code implement into proposed OCDMA-RoF system through optical simulation system. The results revealed that when implementing FCC code, the proposed RoF simulation system has a better performance. The outcomes showed that the FCC code accomplishes long transmission up to $51 \mathrm{~km}$ for bit rate $155 \mathrm{Mb} / \mathrm{s}$ and having minimum BER $1.00 \times 10^{-9}$ for bit rate $622 \mathrm{Mb} / \mathrm{s}$ as compared with RoF system without FCC code. Without any doubt, the maximum out of phase auto-correlation and flexibility in phase cross-correlation values, respectively with different combination of $\left(N, W, \lambda_{\max }\right)$; by increasing $W$ and increasing $\lambda_{\max }$ will improve the system performance with shorter, $N$. This will give an offer in OCDMA-RoF for better quality of service (QoS), fast asynchronous transmission, variable bit rate traffic, higher bandwidth in optical access networks and great potential to be used in the future GB/s wireless access network. Figures and tables, as originals of good quality and well contrasted, are to be in their final form, ready for reproduction. 


\section{ACKNOWLEDGEMENTS}

This work was support by the High Ministry of Education Malaysia under the Fundamental Research Grant Scheme (FRGS) \#9003-00456.

\section{REFERENCES}

[1] Y. Liu, G. Zhang, Q. Li, WDM/TDM hybrid GPON technology, in 2011 Symposium on Photonics and Optoelectronics (SOPO), vol. 2, May 16-18, 2011, pp. 1-3.

[2] G. O. Young, "Synthetic structure of industrial plastics (Book style with paper title and editor)," in Plastics, 2nd ed. vol. 3, J. Peters, Ed. New York: McGraw-Hill, 1964, pp. 15-64.

[3] G. Chang, A. Chowdhury, Z. Jia, et al., Key technologies of WDM-PON for future converged optical broadband access networks, IEEE/OSA J. Opt. Commun. Network. 1 (September (4)) (2009) C35-C50 (Invited).

[4] Svetislav V.Maric, Zoran I. Kostic, Edward L.Titlebaum, "A New family of Optical Code Sequences for Use in Spread-Spectrum Fiber Optic Local Area Networks", IEEE Trans. Comm., vol 41, pp. 1217-1221, August 1993.

[5] J.A. Salehi, "Code division multiple-access techniques in optical fiber network-Part 1: Fundamental principles," IEEE Trans. Commun., vol. 37, pp. 824-833, 1989.

[6] Liu, M.Y.; Tsao, H.W "Multirate Asynchronous Optical CDMA System with Product Code," Communications, SEE Proceedings, Volume: 149 Issue: 56, 2002 Page(s): 299 -304.

[7] Maric, S.V.; Moreno, O.; Corrado, C.J." Multimedia Transmission in Fiber-Optic LANs Using Optical CDMA," Lightwave Technology, Journal of Volume: 14 Issue: 10, Oct 1996 Page(s): 2149 - 2153.

[8] Rashidi, C. B. M., Aljunid, S. A., Ghani, F., Fadhil, H. A., \& Anuar, M. S. (2013). New design of Flexible Cross Correlation (FCC) code for SAC-OCDMA system. Procedia Engineering, 53, 420-427.

[9] Rashidi, C. B. M., Aljunid, S. A., Ghani, F., Fadhil, H. A., Anuar, M. S., \& Arief, A. R. (2014). Cardinality enrichment of flexible cross correlation (FCC) code for SAC-OCDMA system by alleviation interference scheme (AIS). Optik - International Journal for Light and Electron Optics, 125(17).

[10] Rashidi, C. B. M., Aljunid, S. A., Ghani, F., Fadhil, H. A., \& Anuar, M. S. (2013). New Design of Flexible Cross Correlation (FCC) Code for SAC-OCDMA System. Procedia Engineering, 53(0), 420-427.

[11] Rashidi, C.B.M., Aljunid, S.A., Ghani, F., Fadhil, H.A., Anuar, M.S. Cardinality enhancement using Flexible Cross Correlation (FCC) code for Spectral Amplitude Coding Optical Code Division Multiple Access systems. - Journal of Applied Sciences Research.

[12] Abd, T., Aljunid, S., Fadhil, H., Ahmad, R., \& Junita, M. (2012). Enhancement of performance of a hybrid SACOCDMA system using dynamic cyclic shift code. Ukrainian Journal of Physical Optics,13(1),12.

[13] Rashidi, C.B. M. (2013). Encoder-Decoder Design for SAC-OCDMA using Flexible Cross Correlation (FCC) Code Algorithms, 166-168.

[14] H. Fathallah, L. A. Rusch, S. LaRochelle, "Passive optical fast frequency-hop CDMA communications system," J. Lightwave Technol. 17, 397-405 (1999).

[15] Li, et al., "Analysis and Simplification of Three-Dimensional Space Vector PWM for Three-Phase Four-Leg Inverters," IEEE Transactions on Industrial Electronics, vol. 58, pp. 450-464, Feb 2011.

[16] R. Arulmozhiyal and K. Baskaran, "Implementation of a Fuzzy PI Controller for Speed Control of Induction Motors Using FPGA," Journal of Power Electronics, vol. 10, pp. 65-71, 2010.

[17] D. Zhang, et al., "Common Mode Circulating Current Control of Interleaved Three-Phase Two-Level VoltageSource Converters with Discontinuous Space-Vector Modulation," 2009 IEEE Energy Conversion Congress and Exposition, Vols 1-6, pp. 3906-3912, 2009.

[18] Z. Yinhai, et al., "A Novel SVPWM Modulation Scheme," in Applied Power Electronics Conference and Exposition, 2009. APEC 2009. Twenty-Fourth Annual IEEE, 2009, pp. 128-131. 